\title{
RNA-Seq Based Transcriptome Analysis of Ethanol Extract of Saffron Protective Effect Against Corticosterone-Induced PC12 Cell Injury
}

\section{Xi Chen}

Beijing Friendship Hospital, Capital University of Medical Sciences

Ting Yang

Capital Medical University School of Basic Medical Sciences

Cong' en Zhang

Beijing Friendship Hospital, Capital University of Medical Sciences

Zhijie Ma ( $\sim 13811647091 @ 163 . c o m)$

Beijing Friendship Hospital, Capital University of Medical Sciences

\section{Research Article}

Keywords: Ethanol extract of saffron, Depression, RNA-seq, MAPK signaling pathway, QPCR

Posted Date: October 5th, 2021

DOI: https://doi.org/10.21203/rs.3.rs-843621/v1

License: (c) (i) This work is licensed under a Creative Commons Attribution 4.0 International License. Read Full License

Version of Record: A version of this preprint was published at BMC Complementary Medicine and Therapies on January 31st, 2022. See the published version at https://doi.org/10.1186/s12906-02203516-1. 


\section{Abstract \\ Background}

Saffron is a traditional Chinese herbal medicine, which is typically used in clinical to regulate anxiety, tension, and other depression-related conditions. The study aimed to explore the neuroprotective effect of ethanol extract of saffron (EES) on corticosterone (CORT)-induced injury in PC12 cells and further explored its potential mechanism.

\section{Methods}

The authenticity of saffron and the active components of EES were identified by a water test and ultraperformance liquid chromatography-time of flight mass spectrometry system. The screening of cytotoxicity for PC12 cells was incubated with EES in different concentrations for $24 \mathrm{~h}$, and the protective efficacy of EES on CORT $(500 \mu \mathrm{M})$ induced PC12 cell injury, cell viability was assessed by Cell Counting Kit-8 (CCK-8) assay. The DEGs of EES-protected PC12 cells were analyzed using the RNA-seq method, and the results were analyzed for GO and KEGG enrichment. The results of RNA-seq were verified by qPCR analysis.

\section{Results}

The saffron was initially identified as authentic in the water test and 10 compounds were identified by UPLC-MS. The results of CCK-8 demonstrated that EES at concentrations above $640 \mu \mathrm{g} / \mathrm{mL}$ exerted a certain cytotoxic effect, and PC12 cells pretreated with EES $(20,40$, and $80 \mu \mathrm{g} / \mathrm{mL})$ significantly reversed the $500 \mu \mathrm{M}$ CORT-induced cell death. RNA-seq analysis showed that EES regulated 246 differential genes, which were mainly enriched in the MAPK signaling pathway. Dusp5, Dusp6, Gadd45b, Gadd45G, and Pdgfc were further validated by qPCR. Experimental data showed that the results of qPCR were consistent with RNA-seq.

\section{Conclusions}

These findings provide an innovative understanding of the molecular mechanism of the protective effect of EES on PC12 cells at the molecular transcription level, and the above molecules may be potential novel targets for antidepressant treatment.

\section{Background}

Depression is a severe mental disorder that afflicts many people, characterized by low spirits, loss of interest in life, and unexplained pain. According to the data of the World Health Organization, there are over 300 million people worldwide suffer from depression[1]. Patients with depression often suffer from 
sadness, fatigue, crying, pessimism, changes in sleep patterns, and lack of pleasant sensation, making depression the most important cause of disability due to the disease[2].

At present, the treatment for depression is oral antidepressants. Selective serotonin reuptake inhibitors (SSRIs) are the most widely used antidepressants in the clinic and are the first-line treatment for depression. However, current antidepressants can cause serious adverse reactions in patients. The most troubling adverse reactions seem to be gastrointestinal problems, sleep disorders, and sexual dysfunction[3]. At the same time, withdrawal symptoms after drug discontinuation can lead to even worse conditions in patients[4].

Crocus sativus $L$. is a perennial herb commonly known as saffron. Saffron has been used as a spice and medicinal material for many years and as a drug for 3,600 years[5]. Notably, saffron and its main components and secondary metabolites have been proved to have antioxidant[6], neuroprotective[7], and antidepressant effects $[8,9]$, which have been preliminarily verified in clinical practice. The current clinical studies show that saffron has extremely low toxicity, which provides a better guarantee for the safety of long-term treatment of depression patients with saffron[10].

Currently, differentiated PC12 cells have been widely used as an in vitro modeling in neurobiological or neuromuscular mechanisms underlying the antidepressant effect of drugs. Correspondingly, we selected corticosterone to induce injury to pc12 cells. It is the major glucocorticoid in rodents[11]. Excessive corticosterone exposure could activate hippocampal negative feedback regulation instructions and inhibit the activity of the hypothalamic-pituitary-adrenal (HPA) axis[12]. In particular, multiple studies have reported that continuous exposure to high concentrations of CORT can lead to pathological hippocampal neuronal damage and induce depressive-like behavior due to HPA feedback dysfunction[13]. Therefore, the coculture of PC12 with CORT is mature in vitro model for studying depression and nerve injury.

RNA-seq is a new technique in transcriptome research. The technology combines an experimental method of transcriptome sequencing and a database information analysis method of a digital gene expression spectrum. RNA-seq technology has the advantages of high sensitivity, high throughput, high precision, and a wide detection range[14]. It can provide a basic perspective on the understanding of genomic organization and regulation, which in turn can provide valuable information on the intracellular state and the impact of changes in the expression of mutated genes on the study of complex diseases. Elucidation of changes in gene expression is fundamental to understanding the cellular response to internal genetic lesions, external stimuli, and changing environmental conditions. consequently, RNA expression profiles are a unique source of biomarkers for the prediction and classification of human diseases[15]. Therefore, RNA-seq contributes to revealing comprehensive and specific changes in the transcriptome and better studying the protective mechanism of the saffron extract on corticosteroneinduced PC12 cell injury.

\section{Methods}

\section{Identification of saffron by water test}


After saffron was immersed in water at room temperature, there would appear obvious physicochemical phenomena, which could be used to identify the authenticity of the variety. The dried stigma of saffron $(0.5 \mathrm{~g})$, identified by Professor Zhao Kuijun from Beijing Friendship Hospital, Capital Medical University, was weighed and floated on the water, and the color change of water and the morphological change of medicinal material was recorded at different time points.

\section{Preparation of sample solutions}

Ethanol extract of saffron (EES) was extracted by referring to the method in the criterion of the Pharmacopoeia of the People's Republic of China, 2015. Specific for, the saffron $(1 \mathrm{~g})$ was accurately weighed, pulverized, added with $10 \mathrm{~mL}$ of $75 \%$ ethanol, then, ultrasonically extracted in an ice bath. After $2 \mathrm{~h}$, the EES Freeze-dried powder was collected by vacuum freeze dryer. All dried samples were powdered to a homogeneous size and sieved through a No. 65 mesh. In each case, $0.1 \mathrm{~g}$ of powder was ultrasonicextracted for $20 \mathrm{~min}$ with $50 \mathrm{~mL}$ of methanol: water (1:1). The supernatant solution was filtered through a $0.2 \mathrm{~mm}$ PTFE membrane filter before being injected into the UPLC system.

\section{Content determination and component identification of ethanol extract of saffron}

Chromatographic experiments were performed as described previously. Briefly, Acquity UPLC system (Waters, Milford, MA) connected to Xevo G2 Q-ToF MS equipped with an electrospray ionization source (Waters, Milford, MA, USA) was performed on an ACQUITY UPLC T3 column $(2.1 \times 100 \mathrm{~mm}, 1.7 \mu \mathrm{m})$ by gradient elution. The column temperature was maintained at $45^{\circ} \mathrm{C}$ and the flow was $0.4 \mathrm{~mL} / \mathrm{min}$. The mobile phase consisted of solution A (water with $0.1 \%$ formic acid) and solution B (acetonitrile with $0.1 \%$ formic acid). Elution was starting at $5 \% \mathrm{~B}$ which was held constant for $1 \mathrm{~min}$, then increased linearly to $100 \%$ from 1 to $16 \mathrm{~min}$, and the injection volume was $5 \mu \mathrm{L}$.

The ESI-Q-TOF-MS detection was operated using the positive (ESI+) ion mode in the mass range of 50$2000 \mathrm{Da}$. The optimized mass spectrometer parameters were set as follows: capillary voltage of $2.5 \mathrm{kV}$, sample cone voltage of $21 \mathrm{~V}$, source and desolvation temperatures were $130^{\circ} \mathrm{C}$ and $350^{\circ} \mathrm{C}$ respectively. cone gas flow of $50 \mathrm{~L} / \mathrm{h}$, desolvation gas flow of $800 \mathrm{~L} / \mathrm{h}$, nitrogen, and argon was used as the cone and collision gases. Data were switched between the low energy $(4 \mathrm{~V})$ and elevated energy (10-55 V) acquisition modes every $0.2 \mathrm{~s}$.

\section{Cell culture}

PC12 cells were obtained from the Shanghai Institute of Biochemistry and Cell Biology (Shanghai, China). They were cultured in RPMI1640 medium (Gibco, Grand Island, NY, USA) supplemented with $5 \%$ fetal bovine serum (HyClone, Logan, UT, USA), $100 \mathrm{mg} / \mathrm{ml}$ streptomycin, and $100 \mathrm{U} / \mathrm{ml}$ penicillin at $37^{\circ} \mathrm{C}$ in a humidified atmosphere with $5 \% \mathrm{CO}_{2}$.

\section{In vitro cytotoxicity of EES}


For the cell experiments, EES was dissolved in water, prepared into a $2 \mathrm{mg} / \mathrm{mL}$ stock solution (calculated based on the concentration of raw medicinal materials). The screening of cytotoxicity for PC12 cells was assessed by Cell Counting Kit-8 (CCK-8) assay kit (Dojindo, Kumamoto, Japan) as previously described.

Briefly, the PC12 cells were plated into a 96-well cell culture cluster at $4 \times 10^{3}$ per well. Then, the cells were treated with EES in different concentrations for $24 \mathrm{~h}$. At mentioned time points, $10 \mu \mathrm{L} \mathrm{CCK}-8$ was added to each sample respectively, and the cells were incubated at $37^{\circ} \mathrm{C}$ for $1 \mathrm{~h}$. With mixing gently to ensure homogeneous distribution of color, the absorbances of solution were measured at $450 \mathrm{~nm}$ performing a microplate reader (Molecular Devices, USA). The cell was calculated using the equation: cell viability $(100 \%)=\left(\mathrm{OD}_{\text {treatment }} / \mathrm{OD}_{\text {control }}\right) \times 100 \%$.

\section{Protective efficacy of EES on corticosterone induced PC12 cell injury}

The PC12 cell culture method was the same as above. After attachment, respectively, cells were incubated with different concentrations $(10,20,40,80,160,320$, and 640$)$ of EES for an additional 24h. To validate the experiments, fluoxetine ( $10 \mu \mathrm{M}$, Sigma-Aldrich, St. Louis, MO, USA), a classical antidepressant, was used as a positive control. Then, cells were coincubated with $500 \mu \mathrm{M}$ of corticosterone (Sigma-Aldrich, St. Louis, MO, USA) for $24 \mathrm{~h}$. Finally, the calculation method of cell viability rate is the same as above. Optimal EES concentrations for use in the following experiments were determined based on preliminary results.

\section{Total RNA isolation, library construction, and mRNA sequencing}

After corresponding treatment, PC12 cells were lysed in TRIzol reagent (Invitrogen, USA) and total RNA was extracted. A total amount of $2 \mu \mathrm{g}$ RNA per sample was used as input material for the RNA sample preparations. Sequencing libraries were generated using NEBNext UltraTM RNA Library Prep Kit for Illumina (NEB, USA) following the manufacturer's recommendations and index codes were added to attribute sequences to each sample. Briefly, mRNA was purified from total RNA using poly-T oligoattached magnetic beads. Fragmentation buffer was added for interrupting mRNA to short fragments. First-strand cDNA was synthesized with random hexamers using the fragments as templates. Then, Buffer, dNTPs, RNase $H$, and DNA polymerase I was added to synthesize the second-strand cDNA. The double-stranded cDNA was then purified, end-repaired, and A-tailed was added for adaptor ligation. DNA fragments of 250-300 bp in length were selected preferentially by purifying the library fragments with the AMPure XP system (Beckman Coulter, Beverly, USA). Finally, PCR amplification was performed to enrich the cDNA libraries, whose quality was assessed on the Agilent Bioanalyzer 2100 system. After cluster generation, the library preparations were sequenced on an Illumina Hiseq 4000 platform and paired-end $150 \mathrm{bp}$ reads were generated.

\section{Data analysis of differentially expressed genes (DEGs)}

The DEGs were analyzed using the DESeq R package (version 1.10.1), which has been a routine statistical approach to determine the digital differential expressions of genes using a model assuming the negative binomial distribution of the data. To control the false positive rate, Benjamini and Hochberg's 
approach was used for adjusting the $P$ values, and a $P$ value less than 0.05 was considered to indicate a significant difference.

\section{Functional enrichment analysis}

The DEGs were subjected to enrichment analysis of Gene Ontology (GO) and Kyoto Encyclopedias of Genes and Genomes (KEGG). GO functional enrichment and KEGG pathway enrichment were performed by Metascape (https:// metascape.org)[16].

\section{Confirmation of RNA-seq results with qPCR analysis}

PC12 cells were lysed in TRIzol reagent as above. For PCR analysis, CDNA was Synthesized via FastKing RT Kit (With gDNase) (TIANGEN BIOTECH, Cat: KR116). Amplification and quantitation of PCR were performed using a real-time PCR system with SuperReal PreMix Plus (SYBR Green) (TIANGEN BIOTECH, Cat: FP205). mRNA expression levels were normalized to GAPDH mRNA levels. Fold expression determination, gene-to-GAPDH ratios were determined by using the $2^{-\triangle \Delta C t}$ method. The thermal cycles were performed with an initial denaturation at $95^{\circ} \mathrm{C}$ for $10 \mathrm{~min}$, followed by 40 cycles of $95^{\circ} \mathrm{C}$ for $15 \mathrm{~s}$ and $60^{\circ} \mathrm{C}$ for $1 \mathrm{~min}$.

\section{Statistical analysis}

As detailed above, statistical analysis and graphs were performed with GraphPad prism statistical software (GraphPad Software). Results were demonstrated as mean \pm standard deviation (SD). Statistical significance was determined by one-way analysis of variance followed by Tukey's secondary test for significance. $P<0.05$ was considered to be statistically significant.

\section{Results}

\section{Identification and observation of saffron in water test}

The water test of saffron is a traditional method for authenticity identification. After a few saffrons were gently placed on the water surface, then the golden oily liquid was observed floating on the water surface and multiple golden lines reached the bottom of the beaker from the water surface. After 720 seconds, all the water became a golden yellow clear solution (Fig.1A-1L).

\section{Identification of neuroprotective composition in EES}

Figure. $1 \mathrm{M}$ and $1 \mathrm{~N}$ show that chemical base peak intensity (BPI) chromatogram of key compounds characterization of saffron in positive ion mode and negative ion mode determined by UPLC-QTOF/MS. Following the accurate molecular data, 10 chromatographic peaks have been determined using UPLC-QTOF/HDMS (Figure $1 \mathrm{M}$ and $1 \mathrm{~N}$ ). Identification of compounds was achieved by comparison to mass spectral data in the available literature. The data were presented in Table 1. 
Table 1 Identification of differential compounds based on UPLC-MS

\begin{tabular}{|c|c|c|c|c|c|}
\hline $\begin{array}{l}t_{R} \\
(\min )\end{array}$ & Identified compound & $\begin{array}{l}\text { Mass } \\
\text { (neutral) }\end{array}$ & $\begin{array}{l}\text { Error } \\
\text { (ppm) }\end{array}$ & Formulate & $\begin{array}{l}\mathrm{MS}^{\mathrm{E}} \text { fragment ions } \\
(\mathrm{m} / \mathrm{z})\end{array}$ \\
\hline 3.26 & Picrocrocinic acid & 346.1628 & -1.16 & $\mathrm{C}_{16} \mathrm{H}_{26} \mathrm{O}_{8}$ & $345.1546[\mathrm{M}-\mathrm{H}]^{-}$ \\
\hline 3.5 & Astragalin & 448.1006 & 0.00 & $\mathrm{C}_{21} \mathrm{H}_{20} \mathrm{O}_{11}$ & $449.1084[\mathrm{M}+\mathrm{H}]^{+}$ \\
\hline 3.5 & $\begin{array}{l}\text { Kaempferol-3-O-ß-D- } \\
\text { sophorocoside }\end{array}$ & 610.1534 & -3.11 & $\mathrm{C}_{27} \mathrm{H}_{30} \mathrm{O}_{16}$ & $611.1593[\mathrm{M}+\mathrm{H}]^{+}$ \\
\hline 4.12 & Rutin & 610.1534 & 7.04 & $\mathrm{C}_{27} \mathrm{H}_{30} \mathrm{O}_{16}$ & $611.1655[\mathrm{M}+\mathrm{H}]^{+}$ \\
\hline 4.57 & Quercetin-3-0-sophoroside & 626.1483 & 5.44 & $\mathrm{C}_{27} \mathrm{H}_{30} \mathrm{O}_{17}$ & $625.1439[\mathrm{M}-\mathrm{H}]^{-}$ \\
\hline 4.76 & Safranal & 150.1045 & 5.96 & $\mathrm{C}_{10} \mathrm{H}_{14} \mathrm{O}$ & $151.1132[\mathrm{M}+\mathrm{H}]^{+}$ \\
\hline 4.9 & Kaempferol & 286.0477 & -0.35 & $\mathrm{C}_{15} \mathrm{H}_{10} \mathrm{O}_{6}$ & $287.0554[\mathrm{M}+\mathrm{H}]^{+}$ \\
\hline 5.89 & Crocin I & 976.3788 & -0.82 & $\mathrm{C}_{44} \mathrm{H}_{64} \mathrm{O}_{24}$ & $975.3702[\mathrm{M}-\mathrm{H}]^{-}$ \\
\hline 6.38 & Crocetin & 328.1675 & -0.61 & $\mathrm{C}_{20} \mathrm{H}_{24} \mathrm{O}_{4}$ & $\begin{array}{l}329.1751[\mathrm{M}+\mathrm{H}]^{+}, \\
311.1652\left[\mathrm{M}+\mathrm{H}-\mathrm{H}_{2} \mathrm{O}\right]^{+}\end{array}$ \\
\hline 8.1 & Crocin II & 814.3259 & -2.58 & $\mathrm{C}_{38} \mathrm{H} 54 \mathrm{O}_{19}$ & $815.3160[M-H]^{-}$ \\
\hline
\end{tabular}

\section{Effect of EES on PC12 cell viability}

Compared with the negative control group, different concentrations of EES $(5,10,20,40,80,160,320,640$ $\mu \mathrm{g} / \mathrm{mL}$ ) showed low cytotoxicity to PC12 cells, with cell viability of $95.56 \% \pm 0.54 \%, 92.01 \% \pm 1.96 \%$, $92.66 \% \pm 0.97 \%, 91.97 \% \pm 0.65 \%, 90.85 \% \pm 0.48 \%, 90.47 \% \pm 1.10 \%, 90.83 \% \pm 0.69 \%$, and $89.53 \% \pm 0.48 \%$, respectively, without statistical difference among these groups. The toxicity of $1280,2560,5120,10240$, and $20480 \mu \mathrm{g} / \mathrm{mL}$ EES to PC12 cell began to increase by progressively, correspondingly the cell viability were $77.06 \pm 0.83 \%, 74.46 \pm 4.00 \%, 59.87 \pm 3.84 \%, 43.70 \pm 3.37 \%$, and $40.15 \pm 1.70 \%$ (Fig.2A). The results showed that concentrations below $640 \mu \mathrm{g} / \mathrm{mL}$ are safe doses of EES.

\section{Protective effect of ESS in corticosterone-induced PC12 cell}

As shown in Fig.2B, treatment with $500 \mu \mathrm{M}$ of corticosterone for $24 \mathrm{~h}$ had been shown to cause cytotoxicity in PC12 cells. Nevertheless, different concentrations of EES $(20,40$, and $80 \mu \mathrm{g} / \mathrm{mL})$, significantly increased the cell viability by $70.83 \pm 1.77 \%(P<0.05), 77.28 \pm 9.11 \%(P<0.01)$, and $78.24 \pm$ $3.17 \%(P<0.001)$, respectively. As positive control, $10 \mu \mathrm{M}$ FLUO revealed significant significantly increased the cell viability by $83.702 \pm 2.71 \%(P<0.001)$ under $500 \mu \mathrm{M}$ corticosterone co-incubation. 


\section{EES alters RNA expression profiles in PC12 cell}

To further investigate the molecular mechanisms of EES, RNA expression profiles in PC12 cells were analyzed by high-throughput RNA sequencing technology as introduced above. As shown in Fig.3A and 3B, compared with the NC group, there were 998 DEGs in the Model group, including 413 up-regulated and 585 down-regulated DEGs. Compared with the Model group, there are 246 DEGs in the EES group, including 137 up-regulated and 109 down-regulated DEGs. 138 DEGs were further detailed by plotting DEGs Venn diagrams (Fig.3C) and heatmap (Fig.3D and 3E). 45 DEGs were down-regulated after modeling and up-regulated after EES intervention. Reversely, 93 DEGs were up-regulated after modeling and down-regulated after EES administration.

\section{GO enrichment analysis of the DEGs}

GO functional enrichment analysis was performed for the DEGs identified by RNA-seq. and the 138 DEGs were divided into three groups according to their functions and biological pathways, including the biological processes, cellular components, and molecular functions. The results were shown in Fig. 4A.

\section{KEGG pathway analysis of DEGs}

The 138 DEGs were retrieved by the KEGG database, and the results showed that the DEGs were mainly enriched in the 10 pathways (Fig.4B, p『0.05). KEGG pathway analysis helps provide an in-depth understanding of the biological mechanisms of genes. KEGG analysis showed that two most of the DEGs were enriched in mitogen-activated protein kinase (MAPK) signaling.

\section{Validation of the RNA-seq results by qPCR}

To verify the accuracy and reproducibility of RNA-seq results, five highly expressed DEGs were selected to confirm their expression levels by qPCR. (Fig.5A-5E). The trend of expression changes of these selected genes based on qPCR was similar to those detected by the RNA-seq method, which corroborated the reliability and validity of the RNA-seq technology. The primers' design was shown in Table 2.

Table 2 Primer sequences used for qPCR analysis 


\begin{tabular}{lll} 
Gene name & Forward $\left(5^{\prime}-3^{\prime}\right)$ & Reverse $\left(5^{\prime}-3^{\prime}\right)$ \\
\hline Dusp5 & CACACCGCCGACATTAG & GCTCCTCCTCTGCTTGA \\
\hline Dusp6 & TGCCAAGGACTCTACTAACT & CACCAGGACACCACAGT \\
\hline Gadd45a & CATAACTGTCGGCGTGTA & GGCTCTTGTCGTTCTCC \\
\hline Gadd45b & GAAGAAGAGGAGGATGATATTG & CAGTTCGTGACCAGGAG \\
\hline Gadd45g & GTTGATCCAGGCGTTCT & GGTCCTTCCATGTGTCTT \\
\hline PdgfC & GGCAGATAGACTTGGACAG & CCGTATGGACACAGAGAAG \\
\hline GAPDH & GTTCAACGGCACAGTCA & CTCAGCACCAGCATCAC
\end{tabular}

\section{Discussion}

Depression is one of the most common forms of mental illness and the leading cause of disability in a variety of conditions[17]. Approximately 350 million people worldwide suffer from the anxiety and pain caused by depression[18]. Depression affects the quality of life of patients, caregivers, and family members, and thus increases the cost of public health care annually[19]. However, little is known about the pathogenesis of depression. One of the main reasons is the limited availability of validated depression models. These experiments are related to ethical issues and are costly, laborious, and often produce ambiguous results[20]. In contrast, in vitro assay methods are relatively rapid and less demanding methods for testing the neurotoxicity profiles of chemicals. In vitro techniques for studying neurodegenerative disorders are based primarily on chemically induced rodent or human cell lines, which in turn can reveal fundamental, specific changes to individual cells or related molecular pathways[21].

It is estimated that one in 20 people worldwide suffers from depression[22], and although prevalence varies by region and country, depression is common worldwide. However, timely diagnosis and treatment of patients with depression can play an important role in reducing disability, improving the survival and quality of life of patients, and helping to save financial resources in national health sectors[23]. Classical antidepressants involve the regulation of the serotonergic and norepinephrine systems. However, these antidepressants are unsatisfactory because of their slow onset and side effects that sometimes reduce patient compliance, limiting their efficacy[24]. In contrast, traditional Chinese medicinal herbs are safer and have fewer side effects, and can be considered an effective alternative treatment for depression[25].

Many significant pharmacological effects of saffron have been recognized by current research, and one of the most confirmed findings on the benefit of saffron is its antidepressant activity. Clinical trials have been conducted to evaluate the efficacy of saffron in the treatment of mild to moderate depression. The study reported that saffron was more effective than placebo, at least equivalent to the therapeutic doses of imipramine and fluoxetine[26]. Saffron, a perennial herb of the Iridaceae family, is known originally prevalent as a spice. However, as a drug, the chemistry of saffron is complicated; this spice has primary metabolites, which are ubiquitous in nature, such as carbohydrates, minerals, fats, vitamins, amino acids, 
and proteins. A large number of compounds belonging to different classes of secondary metabolites, products of metabolism not ubiquitous but essential for the development or reproduction of the organism, such as carotenoids, monoterpenes, and flavonoids, including main anthocyanins[27].

The exact mechanism of saffron in the treatment of depression is still unclear. We performed highthroughput screening for its potency using transcriptomics and validated the transcription levels of several signature differential genes. Through KEGG pathway enrichment analysis, we found that the DEGs were chiefly enriched in the pathway of MAPK signaling pathway. MAPK superfamily is a cellmediated signaling pathway, which connects inputs to cells, and changes in gene expression led to the modifications in cell phenotype. To mediate its function, the MAPK signaling pathway consists of three kinases, each of which consists of multiple members, namely, extracellular regulated kinases (ERKs), Jun amino-terminal kinase/stress-activated kinase (JNKs/ SAPKs), and p38 kinase. The MAPK signaling pathway is involved in such processes as gene transcription, mRNA translation, protein stability, protein localization, and enzyme activity, thus regulating cell proliferation, cell differentiation, cell survival, and cell death[28]. Markedly, the MAPK pathway has been shown to play a pivotal role in depression and antidepressants.[28, 29]

The largest histone phosphatase that specifically regulates MAPK activity in mammalian cells is the Dual-specificity phosphatases (DUSPs) family phosphatase.[30] DUSPs constitute a large heterogeneous subgroup of the type I cysteine-based protein-tyrosine phosphatase superfamily. DUSPs are characterized by their ability to dephosphorylate both tyrosine and serine/threonine residues. The protein encoded by this gene is a member of the dual-specificity protein phosphatase subfamily. These phosphatases inactivate their target kinases by dephosphorylating both the phosphoserine/threonine and phosphotyrosine residues. They negatively regulate members of the MAPK superfamily (MAPK/ERK, SAPK/JNK, p38), which are associated with cellular proliferation and differentiation. Different members of the family of dual specificity phosphatases show distinct substrate specificities for various MAP kinases, different tissue distribution and subcellular localization, and different modes of inducibility of their expression by extracellular stimuli. [31] Respectively,DUSP5 and DUSP6 inactivate ERK1 and ERK2. DUSP5 is expressed in a variety of tissues with the highest levels in the pancreas and brain and is localized in the nucleus. DUSP6 is expressed in a variety of tissues with the highest levels in the heart and pancreas, and unlike most other members of this family, is localized in the cytoplasm. Mutations in this gene have been associated with congenital hypogonadotropic hypogonadism. Alternatively, spliced transcript variants have been found for this gene.[32]

The growth arrest and DNA damage-inducible protein GADD45 (Gadd45) protein is involved in several cellular mechanisms, including cell cycle control, DNA damage sensation and repair, genotoxic stress, oncology, and molecular epigenetics. These include early and postnatal development, injury, cancer, memory, aging, neurodegenerative diseases, and psychiatric conditions. These proteins act through a variety of molecular signal cascades and are important constituent members of the MAPK signaling pathway. [33] Both GADD45B gene and GADD45G gene is the member of a group of genes whose transcript levels are increased following stressful growth arrest conditions and treatment with DNA- 
damaging agents. The genes in this group respond to environmental stresses by mediating activation of the $\mathrm{p} 38 / \mathrm{JNK}$ pathway. This activation is mediated between their proteins binding and activating MTK1/MEKK4 kinase, an upstream activator of both p38 and JNK MAPKs. The function of these genes or their protein products is involved in the regulation of growth and apoptosis. These genes are regulated by different mechanisms, but they are often coordinated expressed and can function cooperatively in inhibiting cell growth.[34] Ma et al.[35] identified GADD45B as a neural activity-induced immediate early gene in mature hippocampal neurons. Mice with Gadd45b deletion exhibited specific deficits in a neural activity-induced proliferation of neural progenitors and dendritic growth of newborn neurons in the adult hippocampus. Mechanistically, GADD45B is required for activity-induced DNA demethylation of specific promoters and expression of corresponding genes critical for adult neurogenesis, including brain-derived neurotrophic factor (BDNF) and fibroblast growth factor (FGF). Thus, Ma et al. concluded that GADD45B links neuronal circuit activity to epigenetic DNA modification and expression of secreted factors in mature neurons for extrinsic modulation of neurogenesis in the adult brain. Zhang et al.[36] found that the mRNA expression of the GADD45G gene is significantly different between normal pituitary tissue and clinically nonfunctioning pituitary adenomas using CDNA-representational difference analysis. The authors concluded that GADD45G is a powerful growth suppressor controlling pituitary cell proliferation and that it represents the first identified gene whose expression is lost in the majority of pituitary tumors.

Platelet-derived growth factor (PDGF)-induced DNA synthesis and proliferation involves activation of Ras and MAPK. Cross-talk between protein kinase A (PKA) signaling and tyrosine-kinase receptor signaling results in PKA inhibition of the MAP kinase cascade, probably at the level of Raf.[37] The protein encoded by this gene is a member of the platelet-derived growth factor family. The four members of this family are mitogenic factors for cells of mesenchymal origin and are characterized by a core motif of eight cysteines. This gene product appears to form only homodimers. It differs from the platelet-derived growth factor-alpha and beta polypeptides in having an unusual N-terminal domain, the CUB (complement $\mathrm{C} 1 \mathrm{r} / \mathrm{C} 1 \mathrm{~s}$, Uegf, Bmp1) domain. Alternatively, spliced transcript variants have been found for this gene.[38] Fredriksson et al.[38] found that tissue plasminogen activator (tPA), a serine protease, specifically cleaved and activated latent PDGFCC by interacting with the PDGF/ vascular endothelial growth factor (VEGF)like growth factor domain. The growth of primary fibroblasts in culture was dependent on tPA-mediated cleavage of latent PDGFCC, which generated a growth stimulatory loop. Su et al.[39] demonstrated that intraventricular administration of tPA in mice increased cerebrovascular permeability via activation of Pdgfcc and its receptor Pdgfra. Morphologic changes were observed primarily in arterioles.

Immunohistochemical studies showed that Pdgfcc was localized to arterioles in the cortex, striatum, and hippocampus, as well as additional brain regions, and closely resembled tissue distribution of tPA. The Pdgfra receptor was located primarily on perivascular astrocytes associated with arterioles. In a mouse model of ischemic stroke with TPA administration, treatment with the PDGFR-alpha inhibitor imatinib resulted in decreased cerebrovascular permeability and reduced lesion volume. Su et al. suggested that the known association of hemorrhagic complications in some patients with ischemic stroke treated with tPA may be due in part to the activation of PDGFCC by therapeutic tPA. The findings also indicated that PDGF signaling regulates blood-brain barrier permeability. 
Although we have made a preliminary discussion on the mechanism of protection of PC12 cells by EES, our study still stops at the level of in vitro, which can only give partial hint for the changes of biological function. Consequently, next, we will use animal models to further verify the efficacy and conduct an indepth study on the expression level of protein, the executor of biological function.

\section{Conclusions}

In summary, we have found in this study that EES can improve CORT-induced PC12 cell injury, while accompanied by significant changes in RNA expression in PC12 cells. Among them, MAPK pathway genes Dusp5, Dusp6, Gadd45b, Gadd45g, and Pdgfc have undergone noticeable changes. This study helps to understand the potential mechanism of depression, as the damage to neuronal cell survival and neurite growth caused by CORT undoubtedly adversely affects neuronal cell function and signal transduction. Therefore, they may be potential molecular targets of EES in the treatment of depression, providing valuable insights into the treatment or diagnosis of depression.

\section{Abbreviations}

\section{EES}

ethanol extract of saffron; CORT:corticosterone; SSRIs:selective serotonin reuptake inhibitors; HPA:hypothalamic-pituitary-adrenal; CCK-8:Cell Counting Kit-8; DEGs:differentially expressed genes; GO:Gene Ontology; KEGG:Kyoto Encyclopedias of Genes and Genomes; SD:standard deviation; MAPK:mitogen-activated protein kinase; ERKs:extracellular regulated kinases; JNKs/ SAPKs:Jun aminoterminal kinase/stress-activated kinase; DUSPs:Dual-specificity phosphatases; Gadd45:Growth arrest and DNA damage-inducible protein GADD45; BDNF:brain-derived neurotrophic factor; FGF:fibroblast growth factor; PDGF:Platelet-derived growth factor; PKA:protein kinase A; CUB:complement C1r/C1s, Uegf, Bmp1; tPA:tissue plasminogen activator; VEGF:Vascular endothelial growth factor.

\section{Declarations}

\section{Ethics approval and consent to participate}

Not applicable.

\section{Consent for publication}

Not applicable.

\section{Availability of data and materials}

The datasets generated and analyzed during the current study are available in the Metascape repository [https://metascape.org].

\section{Competing interests}


The authors declare that they have no competing interests.

\section{Funding}

This study was supported in part by grants from Research Foundation of Beijing Friendship Hospital, Capital Medical University (No. yyqdkt2018-32).

\section{Authors' contributions}

XC drafted the central idea, analyzed most of the data, and wrote the initial draft of the paper. TY and CN Zhang contributed to refining the ideas, carrying out additional analyses. ZJ Ma revised and finalized this paper. All authors read and approved the final manuscript.

\section{Acknowledgements}

The authors would like to thank their colleagues at the Central laboratory, Beijing Friendship Hospital Affiliated to the Capital University of Medical Sciences, where most of this work was performed. And we special thanks Xiaodong Wang for the assistance of photography techniques.

\section{References}

1. Friedrich MJ: Depression Is the Leading Cause of Disability Around the World. JAMA 2017, 317(15):1517.

2. Malhi GS, Mann JJ: Depression. LANCET 2018, 392(10161):2299-2312.

3. Jakobsen JC, Katakam KK, Schou A, Hellmuth SG, Stallknecht SE, Leth-Møller K, Iversen M, Banke MB, Petersen IJ, Klingenberg SL et al: Selective serotonin reuptake inhibitors versus placebo in patients with major depressive disorder. A systematic review with meta-analysis and Trial Sequential Analysis. BMC PSYCHIATRY 2017, 17(1):58.

4. Reid S, Barbui C: Long term treatment of depression with selective serotonin reuptake inhibitors and newer antidepressants. BMJ 2010, 340:c1468.

5. Dwyer AV, Whitten DL, Hawrelak JA: Herbal medicines, other than St. John's Wort, in the treatment of depression: a systematic review. Altern Med Rev 2011, 16(1):40-49.

6. Asdaq SM, Inamdar MN: Potential of Crocus sativus (saffron) and its constituent, crocin, as hypolipidemic and antioxidant in rats. App/ Biochem Biotechnol 2010, 162(2):358-372.

7. Ghasemi R, Moosavi M, Zarifkar A, Rastegar K, Maghsoudi N: The Interplay of Akt and ERK in A Toxicity and Insulin-Mediated Protection in Primary Hippocampal Cell Culture. J MOL NEUROSCI 2015, 57(3):325-334.

8. Kell G, Rao A, Beccaria G, Clayton P, Inarejos-García AM, Prodanov M: affron(®) a novel saffron extract (Crocus sativus L.) improves mood in healthy adults over 4 weeks in a double-blind, parallel, randomized, placebo-controlled clinical trial. COMPLEMENT THER MED 2017, 33:58-64. 
9. Siddiqui MJ, Saleh M, Basharuddin S, Zamri S, Mohd NM, Che IM, Binti MNN, Binti MH, Mohd HN, Khatib A: Saffron (Crocus sativus L.): As an Antidepressant. J Pharm Bioallied Sci 2018, 10(4):173180.

10. Orio L, Alen F, Ballesta A, Martin R, Gomez DHR: Antianhedonic and Antidepressant Effects of Affron(®), a Standardized Saffron (Crocus Sativus L.) Extract. MOLECULES 2020, 25(14).

11. Bereshchenko O, Bruscoli S, Riccardi C: Glucocorticoids, Sex Hormones, and Immunity. FRONT IMMUNOL 2018, 9:1332.

12. Hunter RG, Seligsohn M, Rubin TG, Griffiths BB, Ozdemir Y, Pfaff DW, Datson NA, McEwen BS: Stress and corticosteroids regulate rat hippocampal mitochondrial DNA gene expression via the glucocorticoid receptor. Proc Natl Acad Sci U S A 2016, 113(32):9099-9104.

13. Zhang HY, Zhao YN, Wang ZL, Huang YF: Chronic corticosterone exposure reduces hippocampal glycogen level and induces depression-like behavior in mice. J Zhejiang Univ Sci B 2015, 16(1):6269.

14. Wang Z, Gerstein M, Snyder M: RNA-Seq: a revolutionary tool for transcriptomics. NAT REV GENET 2009, 10(1):57-63.

15. Nagalakshmi U, Wang Z, Waern K, Shou C, Raha D, Gerstein M, Snyder M: The transcriptional landscape of the yeast genome defined by RNA sequencing. SCIENCE 2008, 320(5881):1344-1349.

16. Zhou Y, Zhou B, Pache L, Chang M, Khodabakhshi AH, Tanaseichuk O, Benner C, Chanda SK: Metascape provides a biologist-oriented resource for the analysis of systems-level datasets. NAT COMMUN2019, 10(1):1523.

17. Cruz-Pereira JS, Rea K, Nolan YM, O'Leary OF, Dinan TG, Cryan JF: Depression's Unholy Trinity: Dysregulated Stress, Immunity, and the Microbiome. ANNU REV PSYCHOL 2020, 71:49-78.

18. Furtado M, Katzman MA: Examining the role of neuroinflammation in major depression. Psychiatry Res 2015, 229(1-2):27-36.

19. Baquero M, Martín N: Depressive symptoms in neurodegenerative diseases. WORLD J CLIN CASES 2015, 3(8):682-693.

20. Bal-Price A, Pistollato F, Sachana M, Bopp SK, Munn S, Worth A: Strategies to improve the regulatory assessment of developmental neurotoxicity (DNT) using in vitro methods. Toxicol Appl Pharmacol 2018, 354:7-18.

21. Llorens J, Li AA, Ceccatelli S, Sunol C: Strategies and tools for preventing neurotoxicity: to test, to predict and how to do it. NEUROTOXICOLOGY2012, 33(4):796-804.

22. Otte C, Gold SM, Penninx BW, Pariante CM, Etkin A, Fava M, Mohr DC, Schatzberg AF: Major depressive disorder. NAT REV DIS PRIMERS 2016, 2:16065.

23. Whiteford HA, Degenhardt L, Rehm J, Baxter AJ, Ferrari AJ, Erskine HE, Charlson FJ, Norman RE, Flaxman AD, Johns $\mathrm{N}$ et al: Global burden of disease attributable to mental and substance use disorders: findings from the Global Burden of Disease Study 2010. LANCET 2013, 382(9904):15751586. 
24. O'Leary OF, Dinan TG, Cryan JF: Faster, better, stronger: towards new antidepressant therapeutic strategies. EUR J PHARMACOL 2015, 753:32-50.

25. Velehorschi C, Bleau P, Vermani M, Furtado M, Klassen LJ: Understanding the role of adjunctive nonpharmacological therapies in management of the multiple pathways to depression. Psychiatry Res 2014, 220 Suppl 1:S34-S44.

26. Wang Y, Han T, Zhu Y, Zheng CJ, Ming QL, Rahman K, Qin LP: Antidepressant properties of bioactive fractions from the extract of Crocus sativus L. J Nat Med 2010, 64(1):24-30.

27. Bathaie SZ, Farajzade A, Hoshyar R: A review of the chemistry and uses of crocins and crocetin, the carotenoid natural dyes in saffron, with particular emphasis on applications as colorants including their use as biological stains. BIOTECH HISTOCHEM 2014, 89(6):401-411.

28. Dhillon AS, Hagan S, Rath O, Kolch W: MAP kinase signalling pathways in cancer. ONCOGENE 2007, 26(22):3279-3290.

29. Duric V, Banasr M, Licznerski P, Schmidt HD, Stockmeier CA, Simen AA, Newton SS, Duman RS: A negative regulator of MAP kinase causes depressive behavior. NAT MED 2010, 16(11):1328-1332.

30. Caunt CJ, Keyse SM: Dual-specificity MAP kinase phosphatases (MKPs): shaping the outcome of MAP kinase signalling. FEBS J 2013, 280(2):489-504.

31. Patterson KI, Brummer T, O'Brien PM, Daly RJ: Dual-specificity phosphatases: critical regulators with diverse cellular targets. BIOCHEM J 2009, 418(3):475-489.

32. Fagerberg L, Hallstrom BM, Oksvold P, Kampf C, Djureinovic D, Odeberg J, Habuka M, Tahmasebpoor $\mathrm{S}$, Danielsson A, Edlund $\mathrm{K}$ et al: Analysis of the human tissue-specific expression by genome-wide integration of transcriptomics and antibody-based proteomics. MOL CELL PROTEOMICS 2014, 13(2):397-406.

33. Sultan FA, Sweatt JD: The role of the Gadd45 family in the nervous system: a focus on neurodevelopment, neuronal injury, and cognitive neuroepigenetics. ADV EXP MED BIOL 2013, 793:81-119.

34. Liebermann DA, Tront JS, Sha X, Mukherjee K, Mohamed-Hadley A, Hoffman B: Gadd45 stress sensors in malignancy and leukemia. Crit Rev Oncog 2011, 16(1-2):129-140.

35. Ma DK, Jang MH, Guo JU, Kitabatake Y, Chang ML, Pow-Anpongkul N, Flavell RA, Lu B, Ming GL, Song $\mathrm{H}$ : Neuronal activity-induced Gadd45b promotes epigenetic DNA demethylation and adult neurogenesis. SCIENCE 2009, 323(5917):1074-1077.

36. Zhang X, Sun H, Danila DC, Johnson SR, Zhou Y, Swearingen B, Klibanski A: Loss of expression of GADD45 gamma, a growth inhibitory gene, in human pituitary adenomas: implications for tumorigenesis. J Clin Endocrinol Metab 2002, 87(3):1262-1267.

37. Bornfeldt KE, Raines EW, Graves LM, Skinner MP, Krebs EG, Ross R: Platelet-derived growth factor. Distinct signal transduction pathways associated with migration versus proliferation. Ann NY Acad Sci 1995, 766:416-430.

38. Fredriksson L, Li H, Fieber C, Li X, Eriksson U: Tissue plasminogen activator is a potent activator of PDGF-CC. EMBO J 2004, 23(19):3793-3802. 
39. Su EJ, Fredriksson L, Geyer M, Folestad E, Cale J, Andrae J, Gao Y, Pietras K, Mann K, Yepes M et al: Activation of PDGF-CC by tissue plasminogen activator impairs blood-brain barrier integrity during ischemic stroke. NAT MED 2008, 14(7):731-737.

\section{Figures}
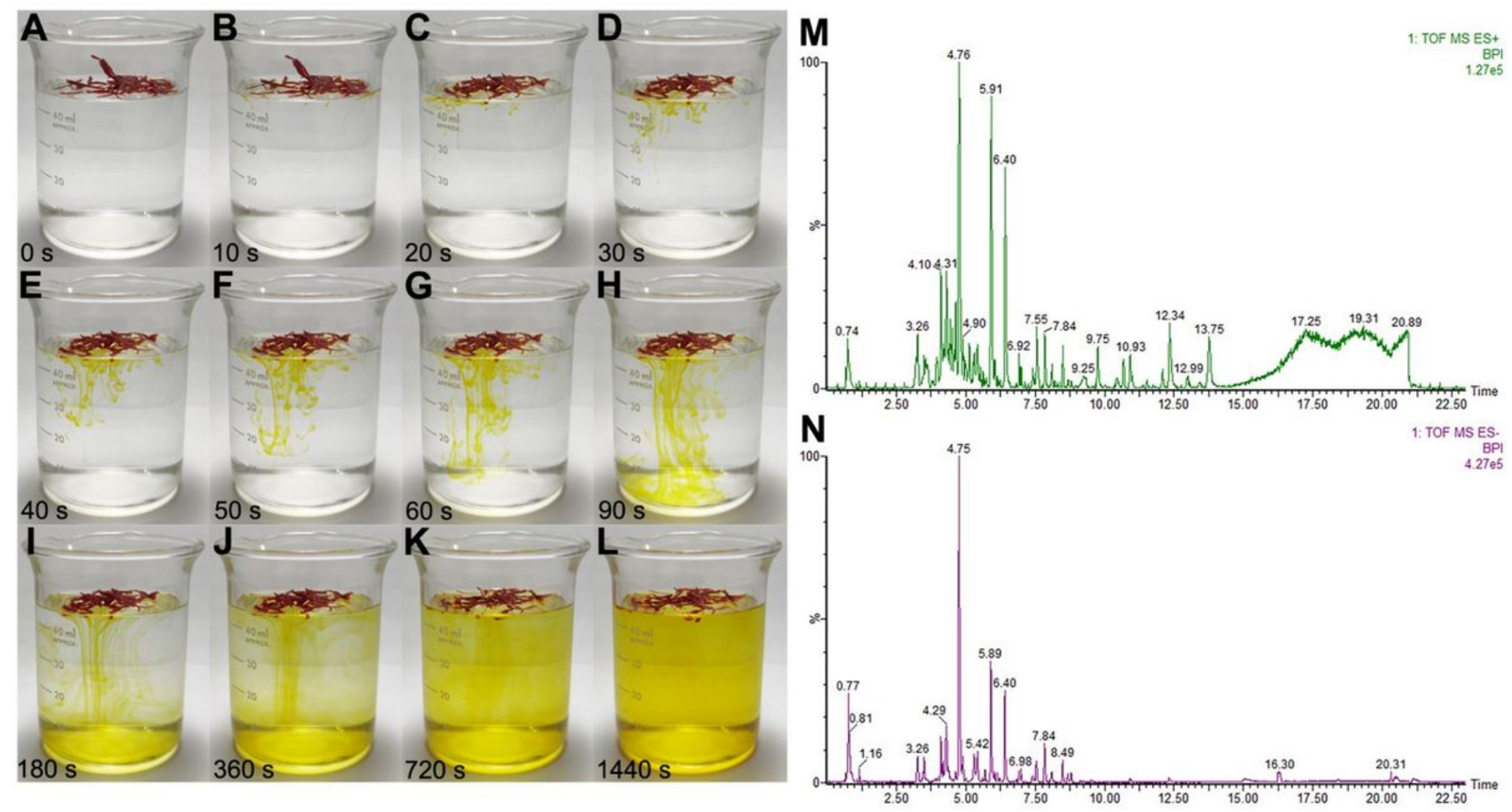

Figure 1

The water test of saffron is a traditional method for authenticity identification. After a few saffrons were gently placed on the water surface, then the golden oily liquid was observed floating on the water surface and multiple golden lines reached the bottom of the beaker from the water surface. After 720 seconds, all the water became a golden yellow clear solution (Fig.1A-1L).

A

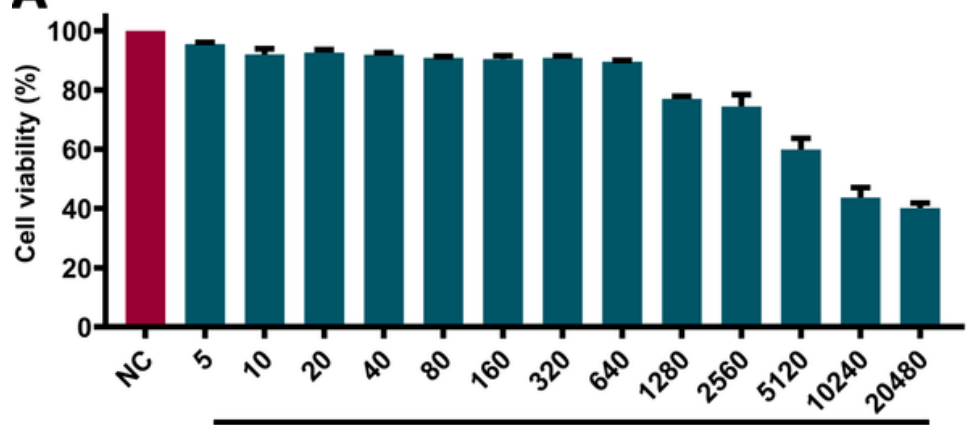

$\operatorname{EES}(\mu \mathrm{g} / \mathrm{mL})$
B

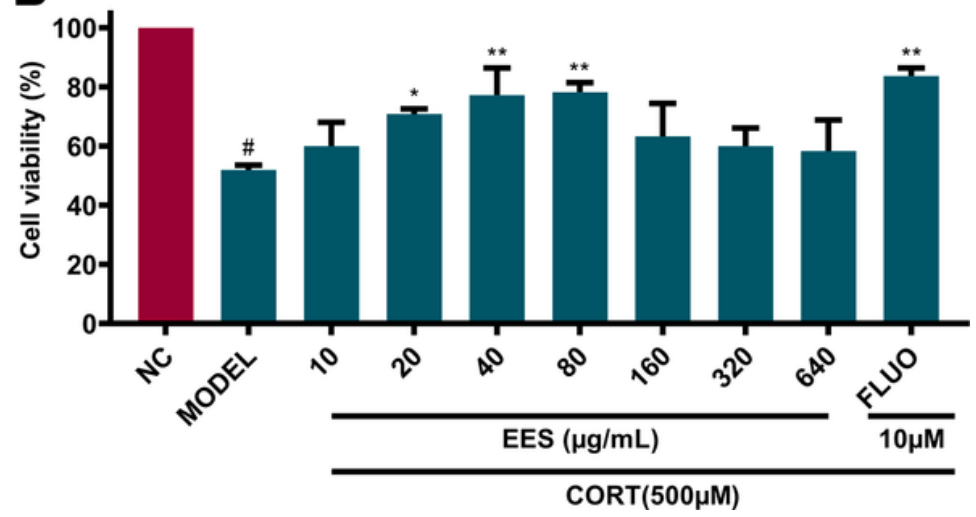

Figure 2 
The toxicity of $1280,2560,5120,10240$, and $20480 \mu \mathrm{g} / \mathrm{mL}$ EES to PC12 cell began to increase by progressively, correspondingly the cell viability were $77.06 \pm 0.83 \%, 74.46 \pm 4.00 \%, 59.87 \pm 3.84 \%, 43.70 \pm$ $3.37 \%$, and $40.15 \pm 1.70 \%$ (Fig.2A). As shown in Fig.2B, treatment with $500 \mu \mathrm{M}$ of corticosterone for $24 \mathrm{~h}$ had been shown to cause cytotoxicity in PC12 cells.
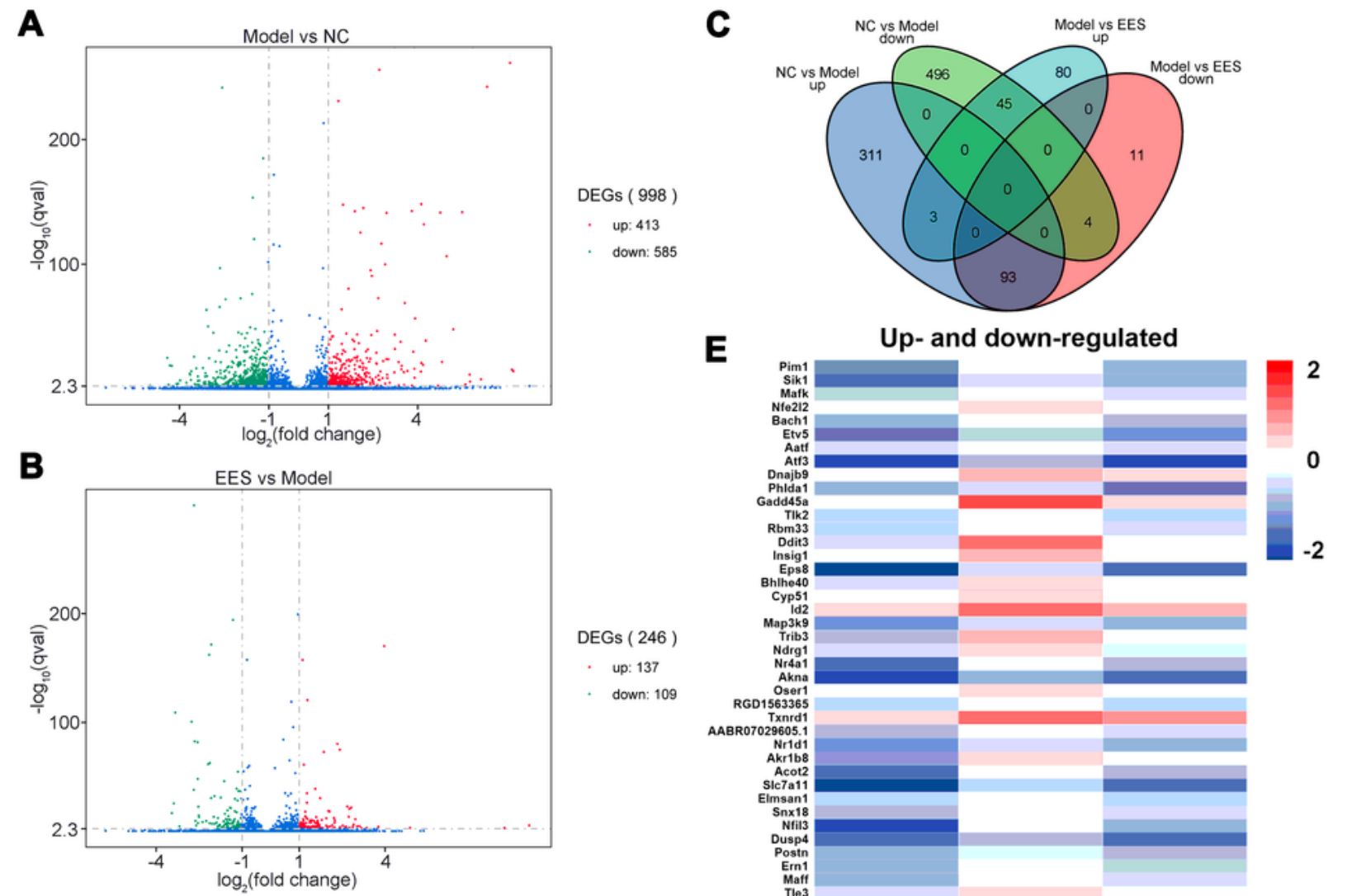

D

DEGs ( 246 )

up: 137

down: 109

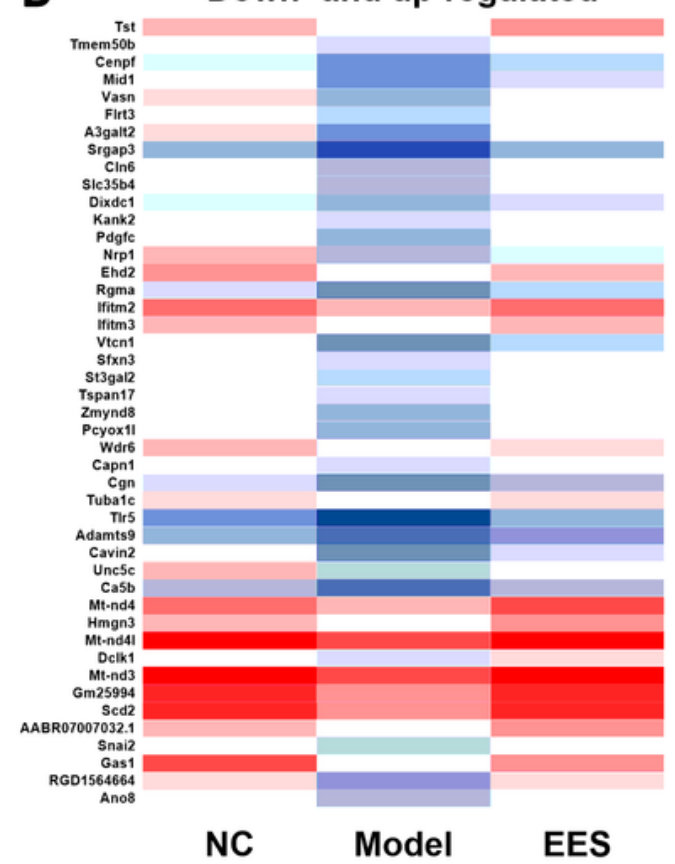

0

$-2$

\section{Figure 3}


As shown in Fig.3A and 3B, compared with the NC group, there were 998 DEGs in the Model group, including 413 up-regulated and 585 down-regulated DEGs. Compared with the Model group, there are 246 DEGs in the EES group, including 137 up-regulated and 109 down-regulated DEGs. 138 DEGs were further detailed by plotting DEGs Venn diagrams (Fig.3C) and heatmap (Fig.3D and 3E).

A

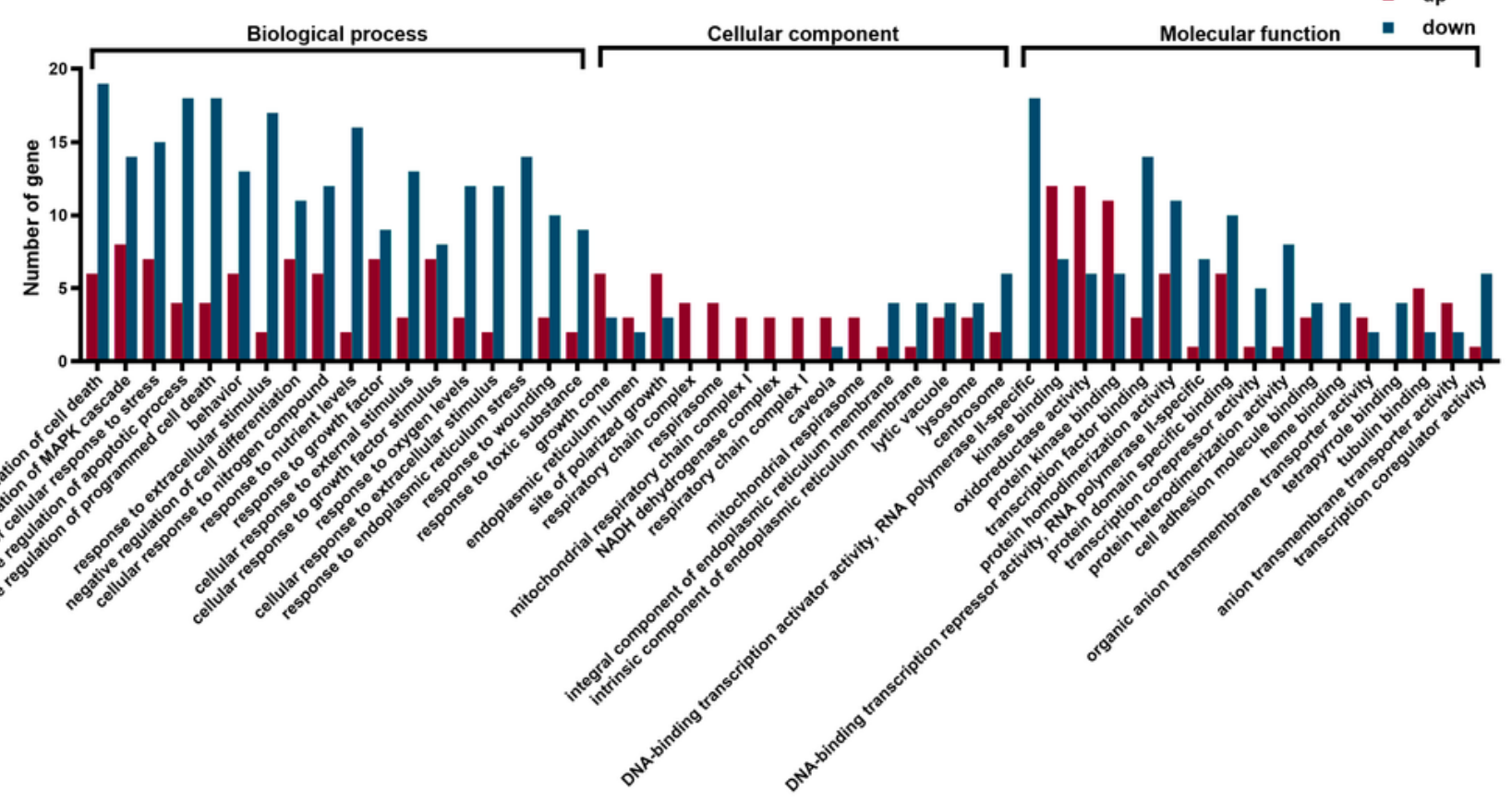

B

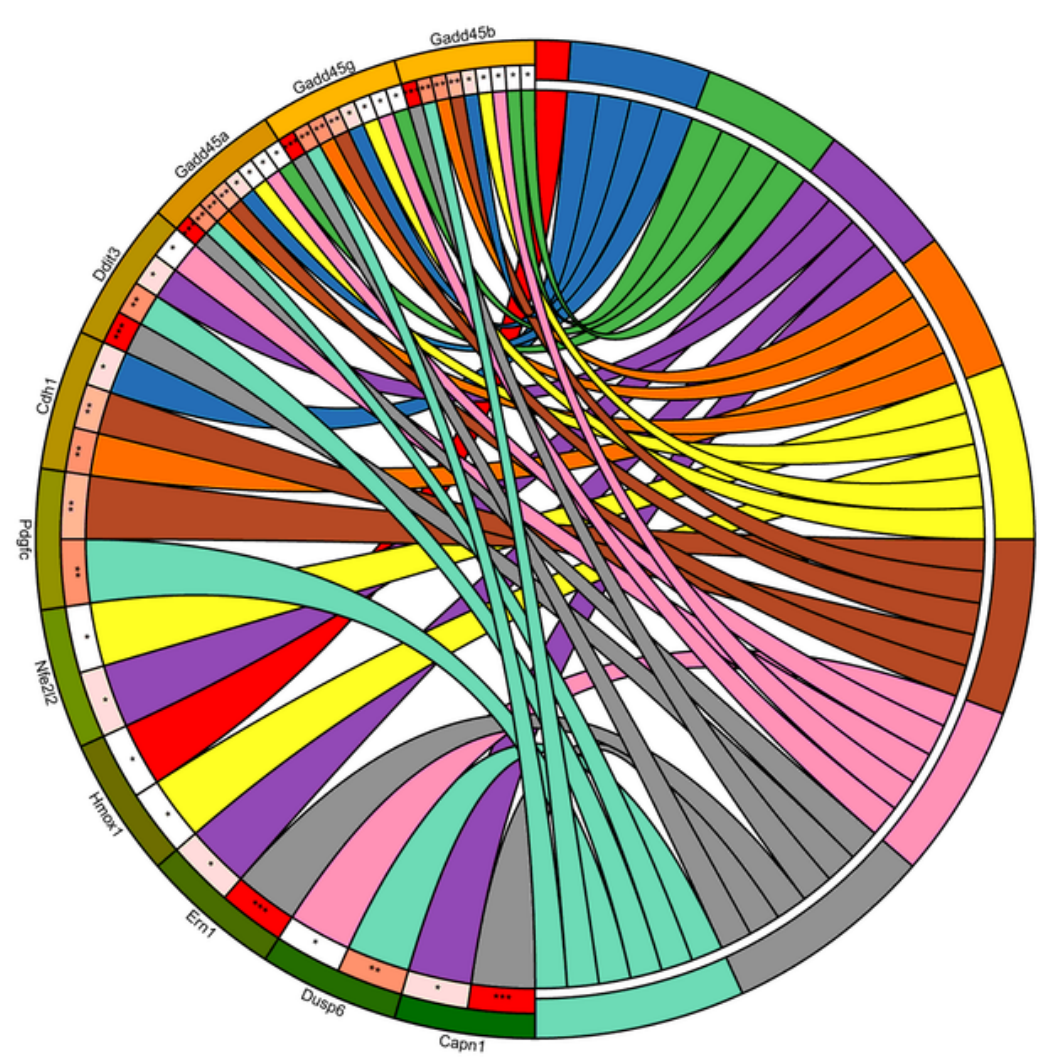

Term

申申Ferroptosis

申 Endometrial cancer

㫗 p53 signaling pathway

申 Protein processing in endoplasmic reticulum 申 Thyroid cancer

Hepatocellular carcinoma

申 Melanoma

- Transcriptional misregulation in cancer 申 Apoptosis

क MAPK signaling pathway

Figure 4 
GO functional enrichment analysis was performed for the DEGs identified by RNA-seq. and the 138 DEGs were divided into three groups according to their functions and biological pathways, including the biological processes, cellular components, and molecular functions. The results were shown in Fig. 4A. The 138 DEGs were retrieved by the KEGG database, and the results showed that the DEGs were mainly

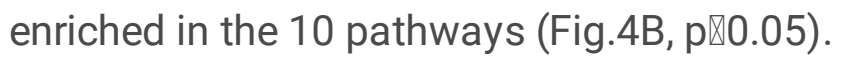

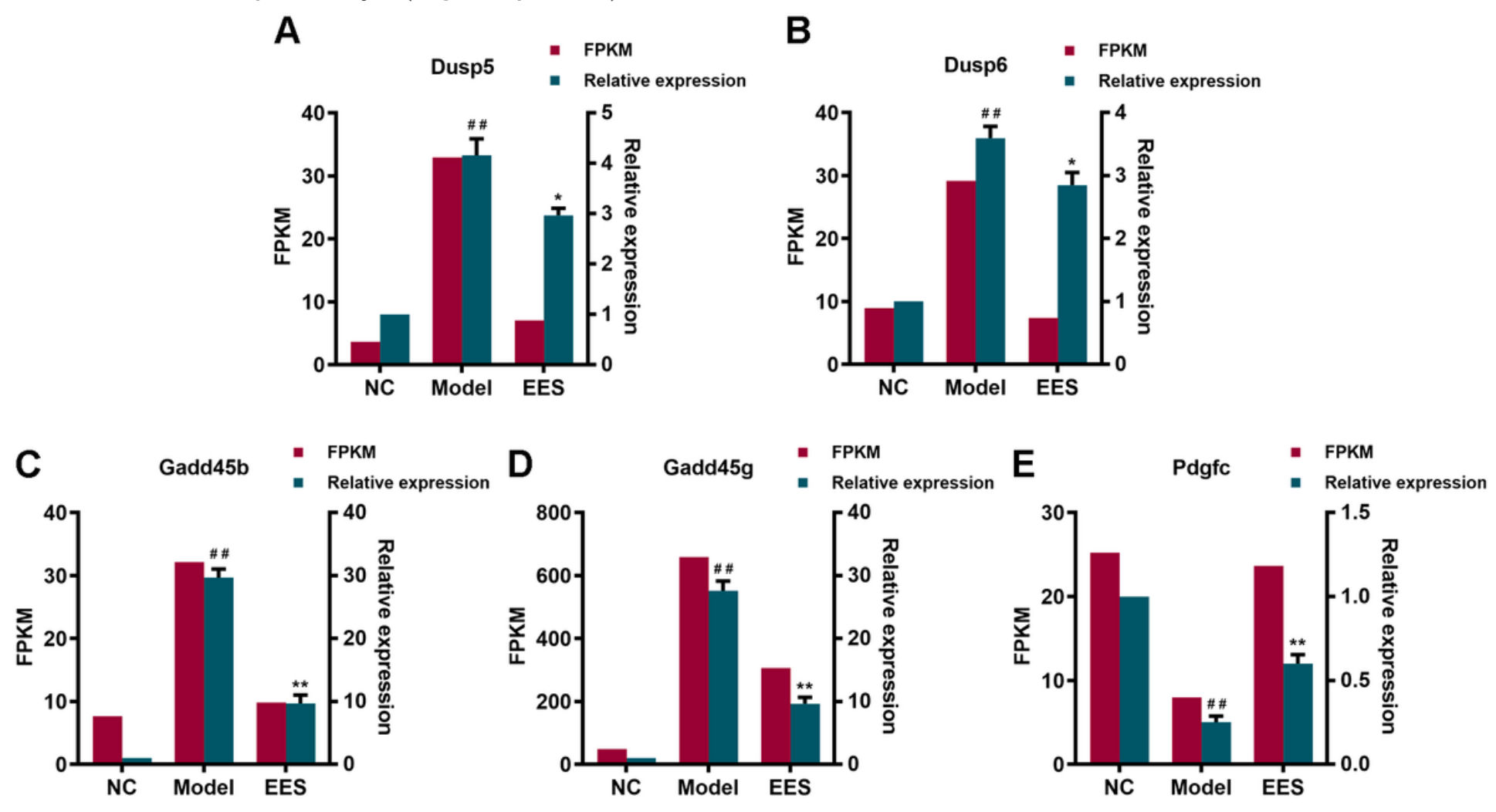

Figure 5

To verify the accuracy and reproducibility of RNA-seq results, five highly expressed DEGs were selected to confirm their expression levels by qPCR. (Fig.5A-5E). 